ISSN 1112-9867

Available online at

http://www.jfas.info

\title{
POLYETHER SULFONE MEMBRANE MODELING AND CONSTRUCTION FOR THE REMOVAL OF NITRATE FROM WATER USING ION INTERFERENCE SULFATE AND IRON NANO-PARTICLE
}

\author{
M. J. Shojaeian and B. Kamayab Moghadas
}

Department of Chemical Engineering, Shiraz Branch, Islamic Azad University, Shiraz, Iran

Published online: 05 June 2016

\begin{abstract}
The aim of this study was constructed the polyether sulfone membrane and modelling it, and for checking impact pressure, the amount of iron nanoparticles and sulfate iron intervention in reducing nitrate irons from water. For the removal ion of nitrate at $50 \mathrm{ppm}$ concentration of nitrate potassium, we made three membranes of 15,18 and 21 percent of sulfone polyether and we studied the effect of pressure of 5, 10, 15 bar without the presence of iron nanoparticles and at the pressure of 10 bar with the presence of 3,6 and 9 percent of iron nanoparticles. At the end by modelling in the proper environment, an appropriate modelling developed for predicting the separation process. In order to analyzing the counfounding sulphate ions with nitrate, the removal of nitrate rate was measured by adding the $50 \mathrm{ppm}$ salt potassium sulfate to the solution containing potassium nitrate and the effect of anion was conducted with the presence of 3 percent of iron nanoparticles and without of it. The results showed that without the presence of iron nanoparticles, most of removal of nitrate rate of 43/2 percent occurred at the pressure of 5 bar and with the membrane of 21 percent. In the next stage using 21 percent of polyether sulfone, the removal of nitrate rate was studied by the presence of 3,6, and 9 percent of iron nanoparticles and at the pressure of 10 bars which the results showed that the highest removal at the presence of 3 percent of iron nanoparticles of 99/42 percent.
\end{abstract}

Author Correspondence, e-mail: Kamyab_bahareh@yahoo.com

doi: http://dx.doi.org/10.4314/jfas.8vi2s.3 
The influence of potassium sulfate without the presence of nano and with the membrane of 21 percent of sulfone polyether was studied with the presence of 3 percent of iron nanoparticles and with the 21 percent of sulfone polyether at the pressure of 5,10 and 15 . The results showed that by addition potassium sulfate to the solution containing potassium nitrate and by increasing the pressure, the amount of nitrate removal has reduced compared to both prior conditions. Finally by using the hole model, laboratory observation was modeled in MATLAB environment, the results of which indicate the ability of the model to predict the behavior of system.

Keywords: separation; nitrate salt; polyether sulfone membrane; modeli.

\section{INTRODUCTION}

Nano-filtration process is more attractive compared to other technologies for the removal of metals and other contaminants, such as reverse osmosis. Nano filtration membranes are between osmosis reverse and ultra-filtration. In other words the separation of the membranes is based on a screening mechanism, while pregnant because of the interactions between membranes and soluble membranes also have an important role in this separation [9].

Nitrate in drinking water are often due to human activities such as excessive use of chemical fertilizers such as potassium nitrate and ammonium nitrate and improper disposal of industrial, human and animal waste.

Nitrate in the water evaporates and remains stable in water. Nitrate is not normally dangerous to health but persistent and excessive consumption can have negative effects on human health, especially children. Nitrate combines with hemoglobin to impaired carry oxygen by the digestive system to become No2 and baby suffered from lack of oxygen and cause failure in newborns [10].

Various methods exist to reduce or eliminate nitrite and nitrate; including dilution, ion exchange, biological, distillation, electro-dialysis, chemical recovery, reverse osmosis, Nano-filtration and Nano-filtration method that was used to remove nitrate [11]. The main objective of this study was to develop a cheaper technology for nitrate removal from water. For this purpose, this study investigates the removal of nitrate from drinking water by Nano filtration to

1. Remove nitrate ions from water.

2. Restore our water for reuse. 
3. Environmental pollution control.

\section{RESEARCH HYPOTHESES}

In this study assumes that:

1. Water content is fixed and does not change.

2. Water flow is constant.

3. Terms of s.s is established.

\section{MATERIALS AND METHODS}

\section{1. materials used for the manufacture of membrane}

Polyether sulfone (PES) N-N-dimethyl acetamide (DMAC) methanol, potassium nitrate, potassium sulphate and zero-valent iron nanoparticles

\section{2. devices and equipment used to manufacture membrane}

1. Dry oven: Heat the oven to eliminate possible moisture in the polymer and additives used. Set the oven temperature is over $80^{\circ} \mathrm{C}$ for 4 to 6 hours of material we put into it.

\section{Scale}

3. Glass maintenance: to provide solutions to make membranes of dark glass medicine have used $30 \mathrm{~mm}$ in size, because it has good stability and its head is securely closed, and prevents the solution possible vaporize.

4. Mixer: mixing set rpm 350 and the mixing time is 24 to 48 hours, stirring with a heater as well, but we do not use heat to build membrance.

5. Ultrasound: Ultrasound devices after eating to reduce and eliminate bubbles in solution are used.

6. Video Cache: Cache is used to smooth the solution prepared from the film. Smoothing operation based on the thickness of 153 micrometers do.

\section{Glass}

8. Coagulation bath: after smoothing solution on the glass so that our membrane is formed in the coagulation bath containing distilled water is put.

9. Pump, 10- EC meters: water EC measurement is done with the Metrohm 827 model.

\section{3-3 membrane manufacturing process}

\subsubsection{Method of making a polymer solution with and without additives}


Initially dishes need washed with distilled water, put in the oven until it is completely sterile and remove any impurities. Selected polymer to 6 hours at $80^{\circ}$ to keep it dry. Then dried polymer weighed, through fitness, we calculate the amount of solvent required. Clean and dry on the human scale, the amount of solvent to be removed. Polyether sulfone are then added to Dymtal Acetamide, close the lid and magnetic stirrer for 48 hours to put on the uniform solution. After the passage of time in order to exit the loss of potential bubbles produced the final solution, ultrasonic devices to ensure full use of the bubbles out of the solution for 30 minutes to put on.

\section{3-3-2 method plumbing Videos}

Intended Solution to enhance the mechanical strength of the membrane separation processes on non-woven polyester is not smooth. Polyester first cut, wash with distilled water, weights and measures glue stuck on the glass so that no bubbles under it.

One of the devices, washed with distilled water and dry thoroughly and use part of it to the numbers put us on the glass, up on solution of glue horizontally throw, in this part of work should be done carefully and vertically stretch film to kill the solution. Speed is very important to do so, after the film draw it immediately with a glass container containing distilled water at ambient temperature put.

\section{3-3-3 phase separation using the coagulation bath}

At this point the solution was separated from the glass-like take on solid form. In about 30 seconds, like a solid membrane made of glass separated.

\section{3-3-4 remove the membrane from the coagulation bath and put them in distilled water.}

In order to remove the final solvent into another container of distilled water transfer membrane will remain in the container for 24 hours. After this time the target membranes with methanol thumbs up data (replacing methanol solvent remaining in the membrane's pores and increases the quality of the membrane and remove moisture from the pores of the membrane.) Membranes for a day put the filter paper. Then put a heavy object on it to be smooth.

\section{3-4 Description of the operating system}

In this study, a cross-flow membrane pilot system using Nano filtration process of Nano filtration was used to separate the nitrate salt. Nano filtration process water or salt water with pressurized water through a membrane permeability of nitrate in the solution are separated. 
Pressure created difference between incoming water pressure and water flow through the membrane impermeable carrier would be.

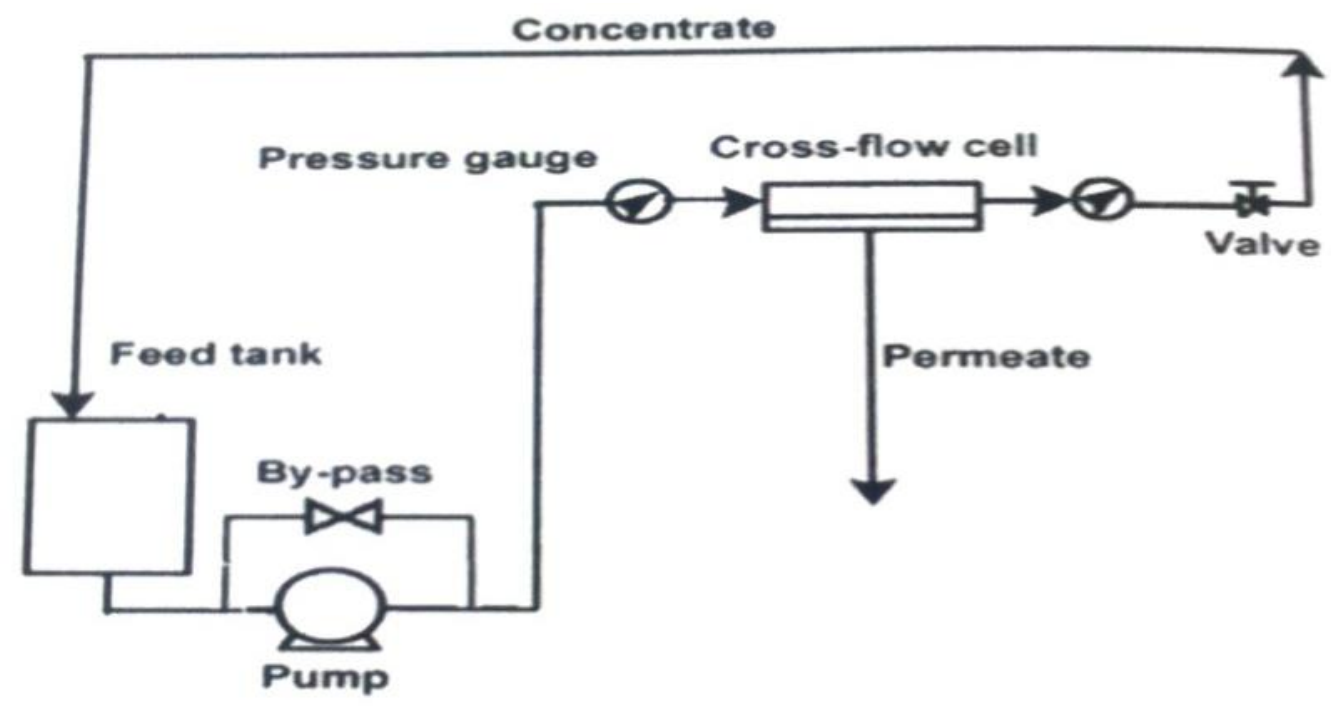

Fig.1. Schematic plan of the process of Nano filtration

We are in the process of Nano filtration in 5, 10 and 15 bar pressure (bar) conducted his experiments that the membrane system includes:

1. Feeding Tank

2. Piston pump

3. Nano-filtration membrane module

4. Two analog pressure gauge

5. Flow control valve is two.

Tank feeding the feed containing the feed stream by the pump from the tank to the polymer membrane conductivity, and two product flows (Article filtered desired) and water comes out of it. Water control valves, inlet and outlet valves before and after the membrane module manually adjustable pressure control the flow of water input and output modules as well. Pressure created by the pump is shown by the barometer. Membrane module made of stainless steel plate and two parts of the membrane separator placed between the two parts and plays their role.

\subsubsection{Membrane processes module}

Membrane modules are always in the chamber of the membrane modules as seating. Modules made in the operation of stainless steel and is rectangular in shape, this module is larger than 
bar10 have high pressures without leaking and operation of the chamber. Cell external dimensions $30 \times 50 \mathrm{~cm}$, a depth of $2 \mathrm{~mm}$ and the width of the channel to $9 \mathrm{~cm}$ is used to calculate the horizontal velocity of the water through the membrane surface used.

\section{3-5 How to separation}

1. Amount of sample aside and throw the rest of the machine. In this case the valve should open the device to burn the pump. Amount of water pressure by the control valve to apply a lot of pressure on the membrane.

2. We will feed the membrane. Material output (filtered) in a clean container collected and analyzed after making standard solutions with the EC meter.

\section{3-6 calculate the flux}

Flux through the membrane can be calculated by the following formula:

$$
\mathrm{J}=\frac{\mathrm{V}}{\mathrm{A} \cdot \Delta \mathrm{t}}
$$

In which the $\mathrm{J}$ flux, $\mathrm{V}$ volume flow, A cross-section of the membrane, $\mathrm{t}$ time is passing.

\section{3-7 Modeling}

MATLAB application was used to modeling in this study.

\section{Conclusion}

\section{4-1 Polyether sulfone layer percent (PES)}

In the first three membranes 15,18 and 21 percent polyether sulfone is made. For each of the membranes made of isolation and amount pressure is measured at different pressures experimental. For this purpose in each test will ppm $50 \mathrm{KNO} 3$ in lit 3 and distilled water for min 40 in the temperature ${ }^{\circ} \mathrm{C} 27$ in the vicinity of each of the membranes separating liquid is then collected and the amount of nitrate by conductivity meter (EC meter) is measured. The results of the effect of polyether sulfone layer at different pressures 5, 10 and 15 times the amount of separation is shown in table 1.

Table 1. Percentage of nitrate salt separation at pressures of 5, 10 and 15 of the membranes 15,18 and 21 percent polyether sulfone

\begin{tabular}{cccc}
\hline \multirow{2}{*}{ Membranes } & \multicolumn{3}{c}{ Pressure (bar) } \\
\cline { 2 - 4 } & 5 & 10 & 15 \\
\hline PES 21\% & 43.2 & 3794 & 30.12 \\
\hline PES 18\% & 20.97 & 17.06 & 14.44 \\
\hline PES 15\% & 7.91 & 5.3 & 1.38 \\
\hline
\end{tabular}




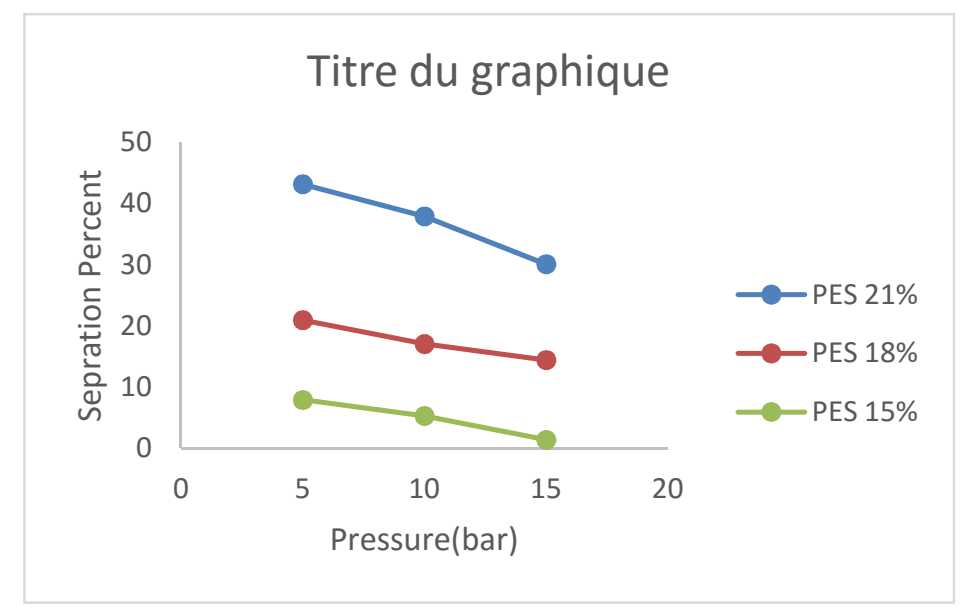

Chart 1. Percent amount of nitrate separation by changing percentage of polyether sulfon layer in different pressures

The results of the effect of polyether sulfone layer at different pressures 5, 10 and 15 times the volumetric flow through are shown in table 2.

Table 2. Volume nitrate salt flux at pressures of 5, 10 and 15 times in the membranes 15,18 and 21 percent polyether sulfone

\begin{tabular}{cccc}
\hline \multirow{2}{*}{ Membranes } & Pressure (bar) & & \\
\cline { 2 - 4 } & 5 & 10 & 15 \\
\hline PES 21\% & 17.31 & 21.54 & 30.3 \\
\hline PES 18\% & 21.64 & 47.61 & 69.26 \\
\hline PES 15\% & 51.94 & 73.59 & 116.88 \\
\hline
\end{tabular}

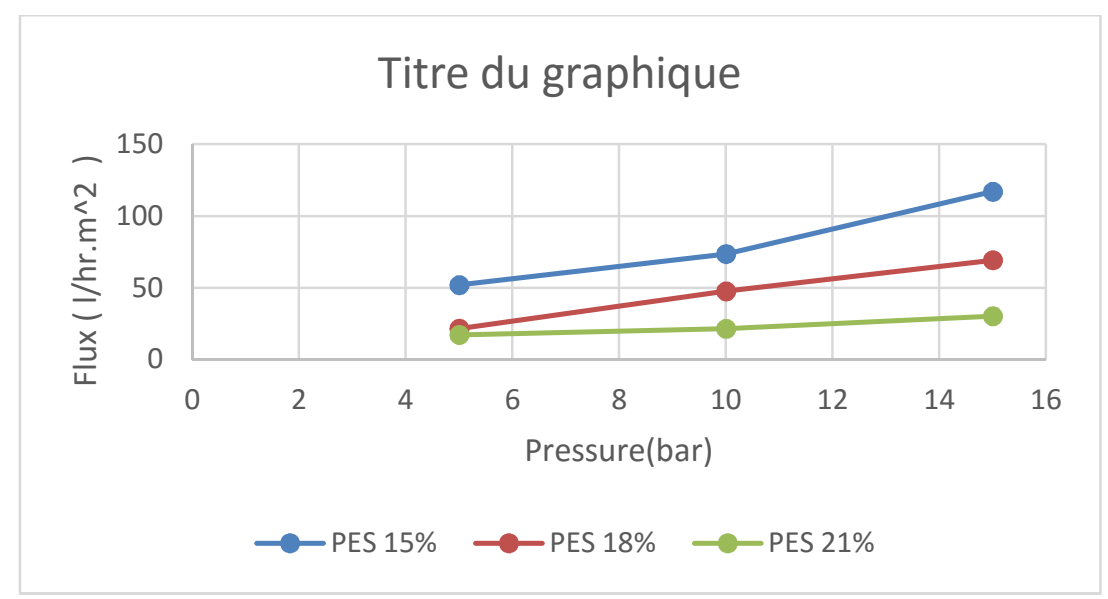

Chart 2. Amount of passing flux by changing percentage of polyether sulfon layer in different pressures 
One of the main reasons for the change of separation membranes made is the effect of membrane pore radius. Increasing the radius leads to increased pore volume and therefore the flux through the membrane material in throughput. As a result, separation is reduced. In this case we can say that cations $\mathrm{k}+$ ionic radius is very small and therefore the low hydration energy, therefore, they can enter the membrane pores in the membrane cavity, some of them remain. (Forces of membrane surface) In this case, the adsorption of particles on the membrane surface forces between them electrostatic and van der Waals forces depends on the process plays an important role. On the other hand NF membranes are negatively charged in this trial, which in turn attract more of these cations in the holes and helps destruction of nitrate ions. With increasing pressure, forces surface remains constant, so amount of passage of ions $\mathrm{K}+$, followed by NO3- (to electrical equilibrium solution) does not change substantially, but because the flux solvent (water) is increased, finally amount of the solution is increased. When this pressure is much greater, flux solvent, self-absorbed cations in the cavities may dislodge and destroy them with the output of nitrates will go to look for them.

\section{4-2 adding iron nanoparticles}

Polyether sulfone different percentages in the last part of amount separation were studied and it was observed that with increasing polyether sulfone from $\% 15$ to $\% 21$, the percentage of separations increases. In other words, the separation of polyether sulfone membrane containing $21 \%$ done. In this section, the effect of iron nanoparticles in the membrane of polyether sulfone $21 \%$ is investigated. For this purpose second series of tests on the membrane and the pressure of 10 bar, for values of 3, 6 and 9 percent were iron nanoparticles. The results of the impact of nanoparticles on amount separation of iron nitrate salt is shown in chart 3.

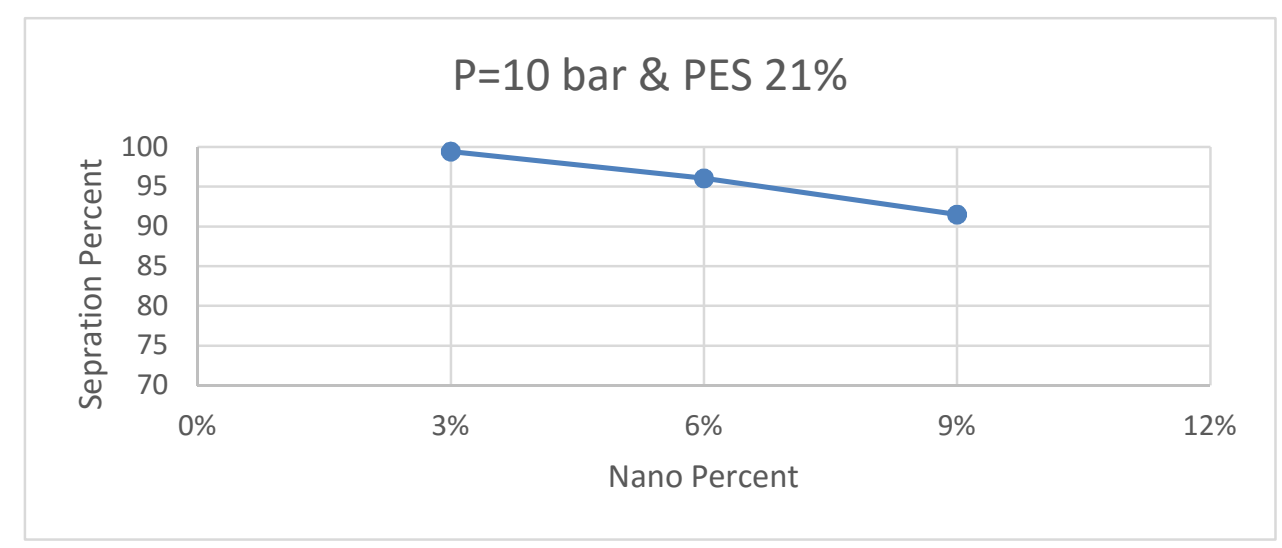

Chart 3. Percent amount of nitrate separation by changing percent. Change of iron nanoparticle in 10 bar pressure and polyether sulfon $21 \%$ 
As can be seen in chart 3, only by adding 3\% iron nanoparticles compared to non-nanoparticle membrane shown in Figure 3, the separation percentage will rise by about 78\%. Because iron mineral surfaces tend to absorb nitrate. Because the use of iron nanoparticles in the membrane is to stabilize nanoparticles in solid structure, either possibility of accumulation and reduce the surface area of nanoparticles will be lost and will increase the surface area of the membrane. Therefore increasing the surface area of the membrane can consume less of these particles, higher levels of nitrate removal and separation of the particles from the environment easily.

Changes flux by increasing the amount the percentage of iron nanoparticles is shown in chart 4.

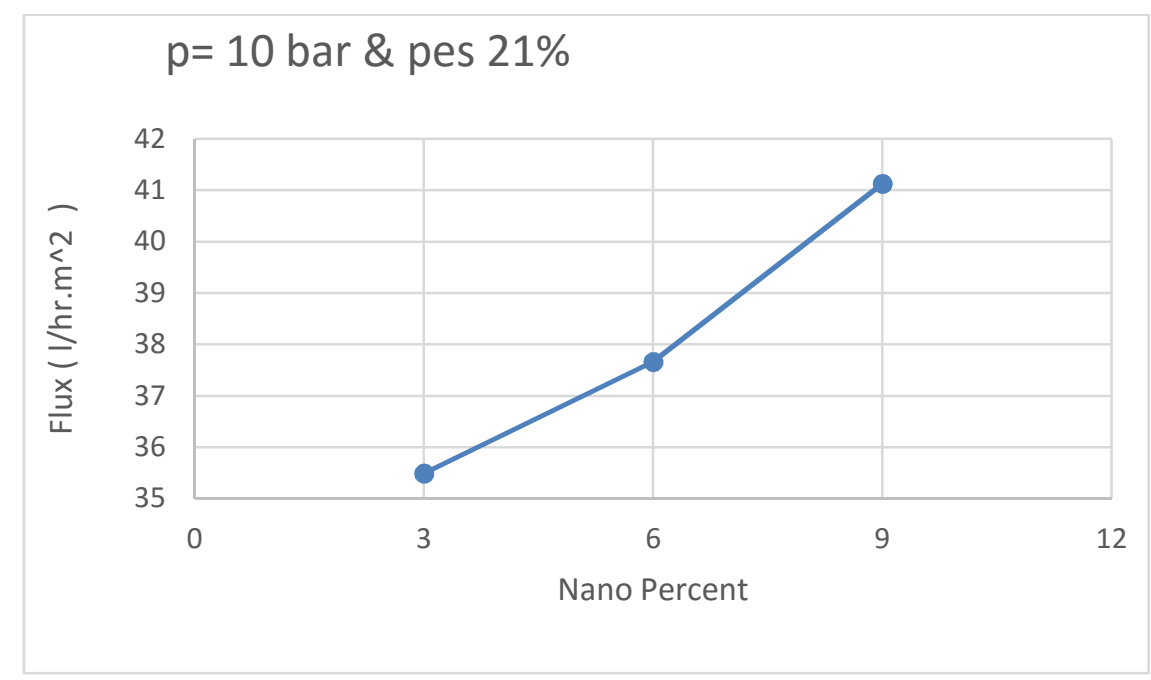

Chart 4. Amount of passing flux by changing percentage of iron nanoparticle in $10 \mathrm{bar}$ pressure and $21 \%$ polyether solfun

The reason for this can be described by saying that the presence of iron nanoparticles and cavity-causing because of the hydrophilic polymer solution to increase the velocity of the solvent and non-solvent in the coagulation bath and as a result, expected channel to increase the size of the holes also been met. The presence of iron nanoparticles in the polymer solution to specific concentrations $(3 \%)$ due to an increase in the velocity of solvent and non-solvent in the coagulation bath to increase the size of the holes and channels, and at higher concentrations $(6 \%)$ The increase in the size of pores and channels is evident and makes it flux more.

\subsection{Polyether sulfone membrane modeling}


Among the models of mass transfer in the membrane, the Capillary model by pore membrane, is the only one that moving parts in the form of two-dimensional. According to this model provided the velocity and flow of current. In this equation $\beta$ convective coupling coefficient (a measure of the pore membrane prevents ions in space) $U_{m}$ mean flow velocity, $C_{i}{ }^{I}$ and $C_{i}{ }^{I I}$ to the ion concentration in the feed and concentration is on the side permeate. Dimensionless parameter $\mathrm{A}_{\mathrm{i}}$ is obtained from equation (4) [2].

$$
\bar{j}_{X, i}=\beta_{i} \frac{U_{m}\left(-C_{i}^{I I}+A_{i} C_{i}^{I}\right)}{A_{i}-1}
$$

Dimensionless parameter $A_{i}$ is obtained from equation (4). In this parameter $D_{i}$ penetration, and $\mathrm{X}$ is the thickness of the membrane [2].

$$
A_{i}=\exp \frac{\Delta X \cdot \beta_{i} \cdot U_{m}}{D_{i}}
$$

$\mathrm{U}_{\mathrm{m}}$ parameter mean flow velocity is obtained from Equation 5 in which $\mathrm{P}$ pressure difference between two sides of the membrane, swapping liquids viscosity, $R_{p}$ membrane pore radius and $\mathrm{D}_{\mathrm{i}}$ is the diffusion coefficient.

$$
U_{m}=R_{P}^{2} \frac{\Delta P}{8 . \mu . \Delta X}
$$

Parameter $\beta$ convective coupling factor depending on the value of $\lambda$ is obtained from equations 6 and 7. $\lambda_{i}$ dimensionless parameter of the radius $\left(r_{i}\right)$ is the membrane pore radius is calculated from equation 4-6. [6]

$$
\begin{gathered}
\beta_{i}=1-2.104 \lambda_{i}+2.09 \lambda_{i}^{3}-0.95 \lambda_{i}^{5} \quad \lambda_{i}<0.22 \\
\beta_{i}=\frac{1}{44.57-416.2 \lambda_{i}+934 \lambda_{i}^{2}+302.4 \lambda_{i}^{3}} \quad \lambda_{i}>0.22 \\
\lambda_{i}=\frac{r_{i}}{R_{p}}
\end{gathered}
$$

Diffusion coefficient parameter is part of the equation $9 \mathrm{k}$ is Boltzmann's constant and $\mathrm{T}$ temperature is obtained in this equation. 


$$
D_{i}=\frac{k T}{6 \pi \cdot \mu \cdot r_{i}}
$$

) 9(

The concentration of each component in the permeation of the equation 10 is obtained in which $\mathrm{C}^{\prime}$ is concentration.

$$
C_{i}^{I I}=\frac{\bar{j}_{i} C^{\prime}}{\sum_{i=1}^{n} \bar{j}_{i}+U_{m} C^{\prime}}
$$

Finally percentage of removal of ions from the equation 11 is obtained. [1 and 2]

$$
R_{i}=\left(1-\frac{C_{i}^{I I}}{C_{i}^{I}}\right) * 100
$$

\section{4-3-1 Capillary model results}

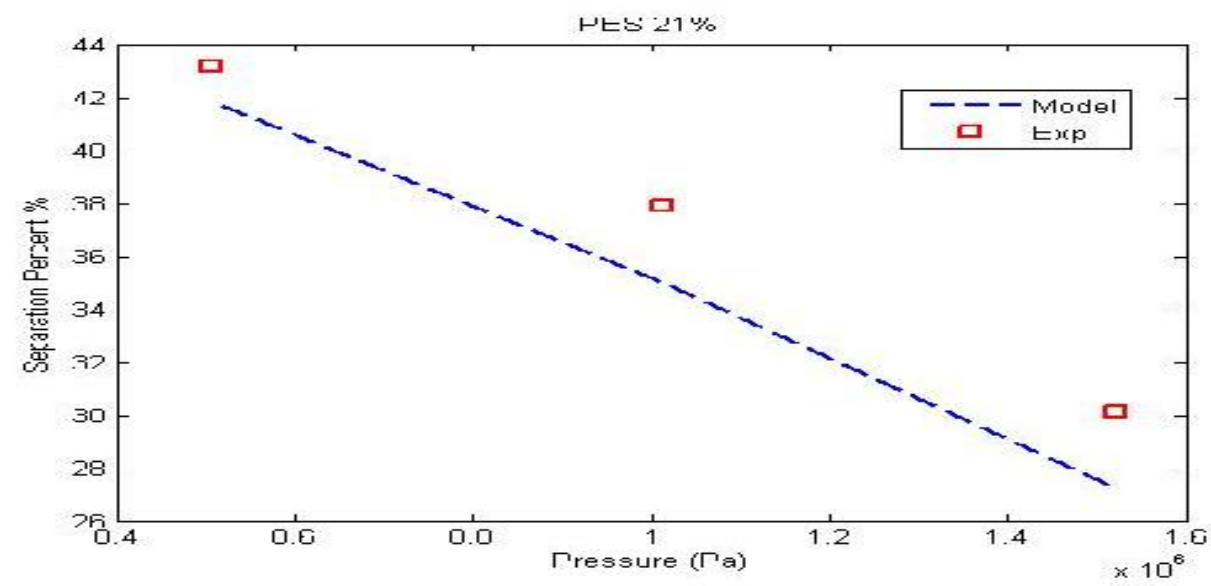

Chart 5: Capillary model and experimental results amount the separation the percentage of polyether sulfone layer $21 \%$ nitrate at different pressures.

As shown in chart 5 results of the modeling process as well as the results of polyether sulfone separation percentage of $21 \%$ of its forecast. You can also use this model to predict the behavior of pressure systems other than the operating pressure. With feed pressure increases, according to equations 3 to 5 flux volume increases, as a result the percentage of separation will be reduced. These changes are well shown in chart 5 . 
Modeling results of related to volumetric flow through the polyether sulfone $21 \%$, with the results shown in Figure 8.

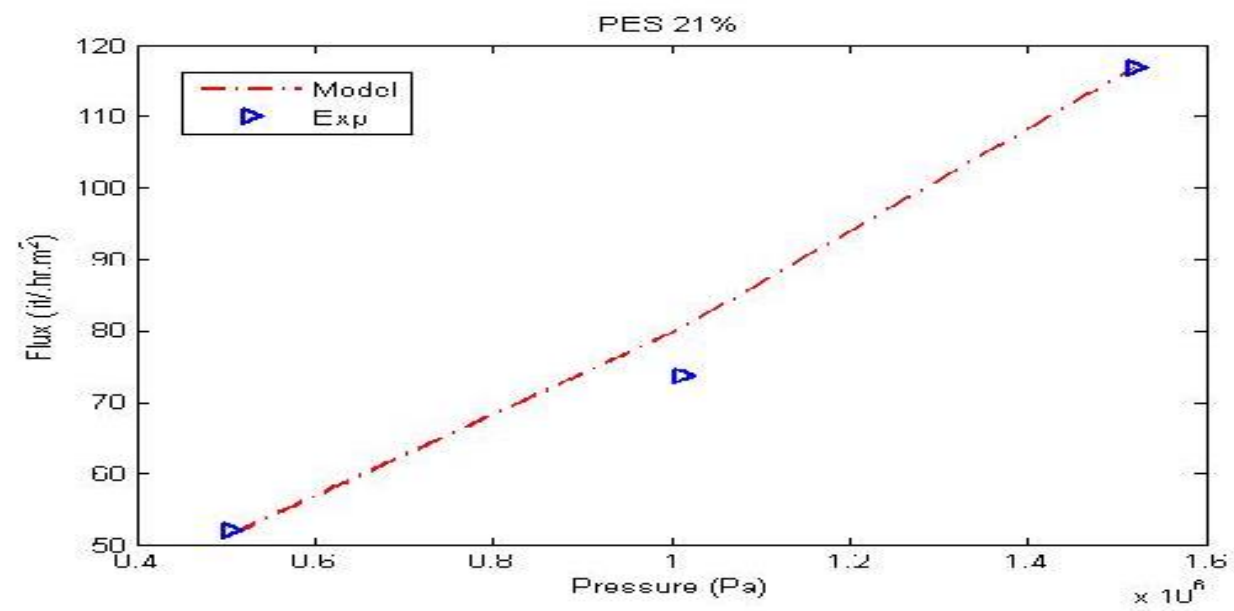

Chart 6. Capillary model and experimental results amount charge polyether sulfone layer $21 \%$ by volume nitrate at different pressures

As can be seen in chart 6 , with increasing pressure difference across the membrane and thus increase the driving force mass transfer, mass flux through the membrane increases. Forecast volumetric flow cavity model with red lines and experimental data points are marked blue in chart. As shown in the results are well matched. It also comes as the chart 4, curve flux provided by the model and the experimental results within the linear trend. This is due to the low initial concentration of salt is evaluated and the resulting concentration polarization. These results with studies of Torabian and colleagues [1] Xu and Lebrun [8] and Paugam and colleagues [7] and correspond well.

In the case of polyether sulfone membrane nanoparticles is added, amount separation of the case without the nanoparticles increases. This is due to facilitate the permeability of the membrane is nitrate. So, as we have seen, amount separation and mass flux through significantly increased. Change approximate diffusion coefficient of nitrate by adding iron nanoparticles were extracted. [3, 4] Capillary model to predict the performance the percentage of the separation process in the presence of Nano-particles is shown in chart 7. 


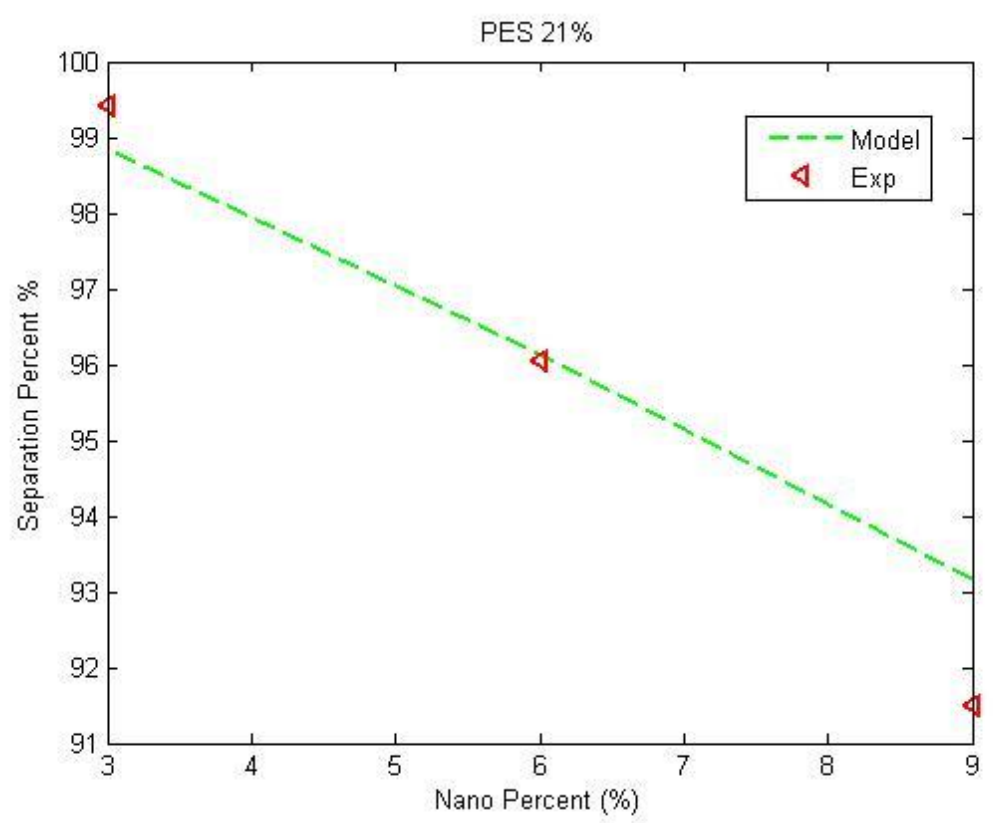

Chart 7. Model Capillary in the layer of polyether sulfone and the results the percentage of the separation of nitrate and $21 \%$ in different percentages of iron nanoparticles

Also the results related to modeling volume flux polyether sulfone $21 \%$ in the presence of nanoparticles is shown in chart 8 .

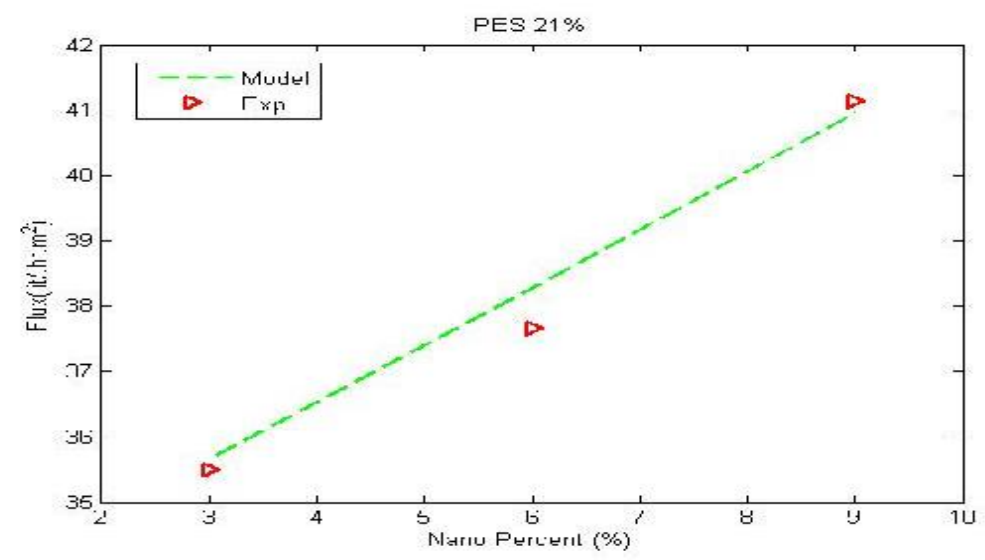

Chart 8: Model Capillary in the layer of polyether sulfone $21 \%$ and laboratory results nitrate flux volume and in different percentages of iron nanoparticles

As can be seen from chart 7 and 8 , a good model is able to predict the experimental results.

\section{4-4 sulphate as a confounding variable}

At this stage the sulfate ion nitrate salt was added to the initial solution and the effect of this intervention on the percentage of isolated ions in both the presence and absence of iron 
nanoparticles in the membrane were examined. For this purpose experiments on membrane of polyether sulfone $21 \%$ pure and polyether sulfone membrane $21 \%$, nanoparticle containing $3 \%$ and in the pressure of 5, 10 and 15 was conducted and the results were listed in Table 3 .

Table 3. Percentage of nitrate salt separation at pressures of 5,10 and 15 times in the membranes of polyether sulfone $(21 \%)$ with and without the presence of nanoparticles

\begin{tabular}{cccc}
\hline Membrane & \multicolumn{3}{c}{ Pressure ) bar( } \\
\cline { 2 - 4 } & 5 & 10 & 15 \\
\hline $\begin{array}{c}\text { PES 21\% } \\
\text { 3\% of iron Nano- } \\
\text { particle }\end{array}$ & 74 & 70.59 & 67.98 \\
\hline $\begin{array}{c}\text { PES 21\% } \\
\text { without iron Nano- } \\
\text { particle }\end{array}$ & 34.011 & 27 & 23.58 \\
\hline
\end{tabular}

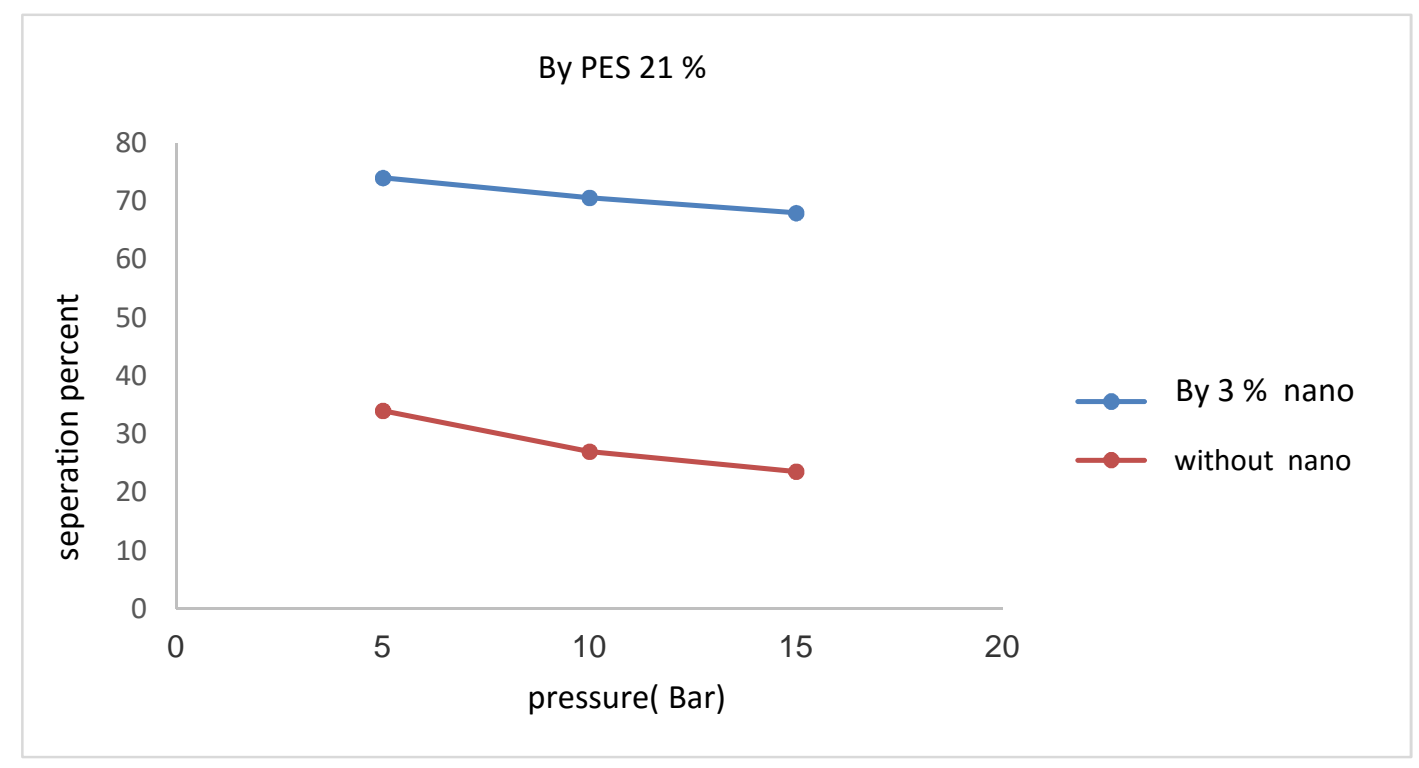

Chart 9. Percent amount of nitrate separation in presence of sulfate accompanied by Nano particle and without it

As can be seen from Table 3 and Chart 9, the presence of sulfate ions in both membrane containing 3\% nanoparticles and nanoparticle without the presence, reduced the percentage of its isolation. As a result of the addition of soluble sulfate, nitrate removal rate decreases. Due 
to the high negatively charged sulfate and nitrate higher hydration energy, is rejected by the membrane and because the solution is electrically neutral stand on the other side of the membrane and thus decrease the isolation. This trend of changes the results obtained by the Torabian [1] that the experimental study of anion and cation with nitrate solution on amount removal pays very well matched.

The results related to the effect of interfering ions on volume flux through the membrane is shown in chart 10 .

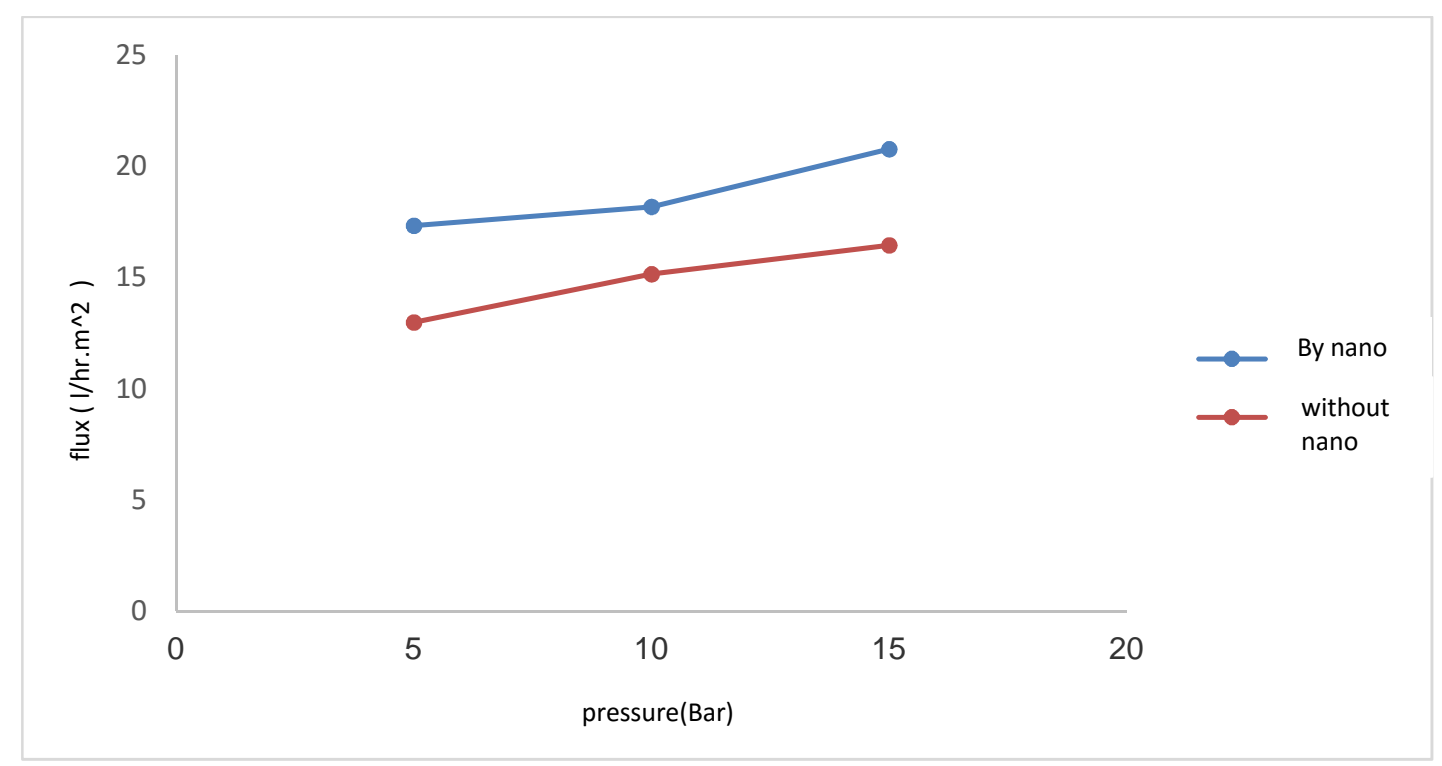

Chart 10. Amount of passing flux in presence of sulfat with nanoparticle and without it

\subsection{The results of various tests on the membrane}

\section{4-5-1 Image SEM:}

Scanning Electron Microscope briefly say SEM, a type of electron microscopes is very popular especially in nanotechnology has found many applications. Initially main advantage of SEM, microscope images directly from a solid sample resolution and better focus and resolution compared with optical microscopy, respectively. But later expanded executive power and operating system and the methods of analysis, such as x-rays to determine the chemical composition and electron channeling was equipped to determine the crystalline state. [5]

SEM images related to membrane of polyether sulfone $21 \%$ and $21 \%$ polyether membrane Solon 3\% nanoparticles shown in Figures 2 and 3. In sample containing iron nanoparticle size and number of channels and cavities will change. Figure 3 weight percent polyether sulfone 
and 3 containing 21 weight percent of iron nanoparticles with a larger size and greater number of cavities in the figure 2 without nanoparticles. When an iron nanoparticle additives are added to the polymer solution, quickly replacing the solvent with water in the shower at the conclusion of phase separation increases. Therefore membrane pore structure becomes more and larger.

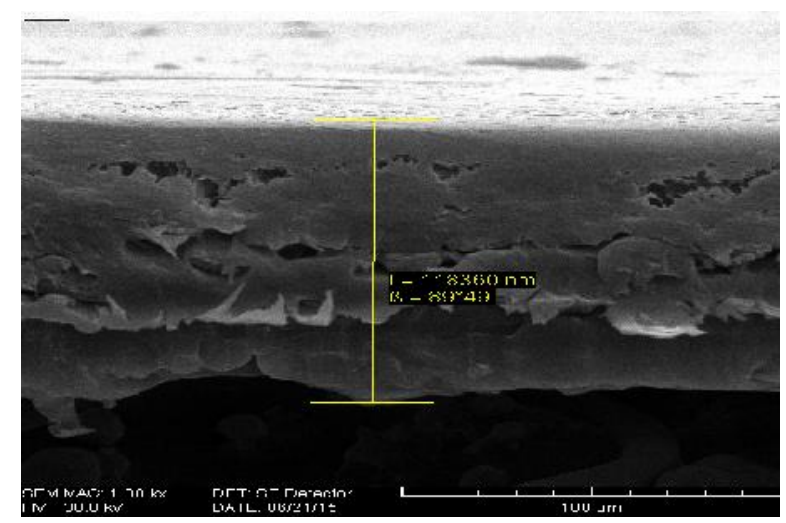

Fig.2. SEM micrograph related to membrane PES 21\%

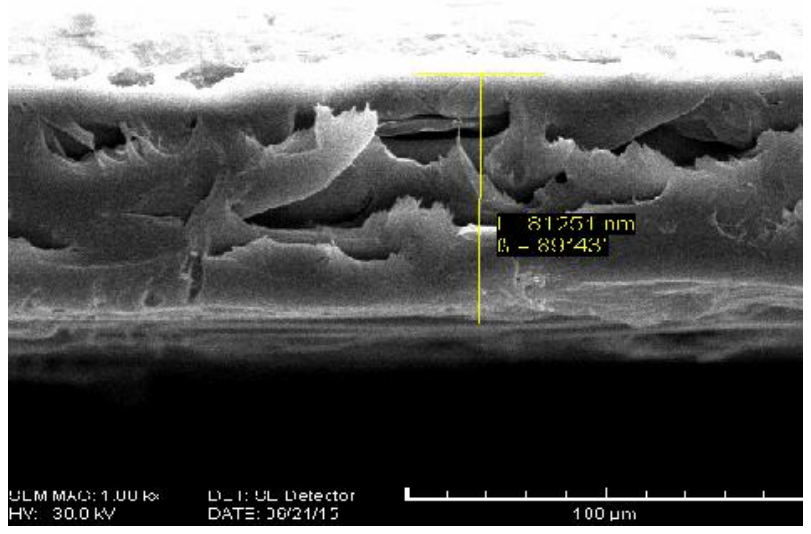

Fig.3. SEM micrograph related to membrane PES $21 \%$ Nano $3 \%$ by

\subsubsection{XRD spectra}

X-ray diffraction (XRD) X-Ray Diffraction model D8, Advance elements are manufactured by Bruker. Radiation used is copper. As is clear from the images that related to nanoparticle is iron in Figure $1544.804=2 \mathrm{Fe}$ we have our 17 correspond to the image. In a state image after 13 to 12 has a peak iron, 0696-06 in accordance with standard card at the point of 44.804 $=2$ we have we Fe that the peak width at half maximum $\mathrm{FWHM}=0.117^{0}=\mathrm{B}$ is the height 
that through this we can use the law scherrer, crystalline grain size in terms of peak width to obtain.

$$
\mathrm{K}=0.9: \lambda=1.54 \mathrm{~A}
$$

)formula 3(

$$
\text { average of particle size } D=\frac{\mathrm{K} \lambda}{\mathrm{B} \cos \theta}
$$

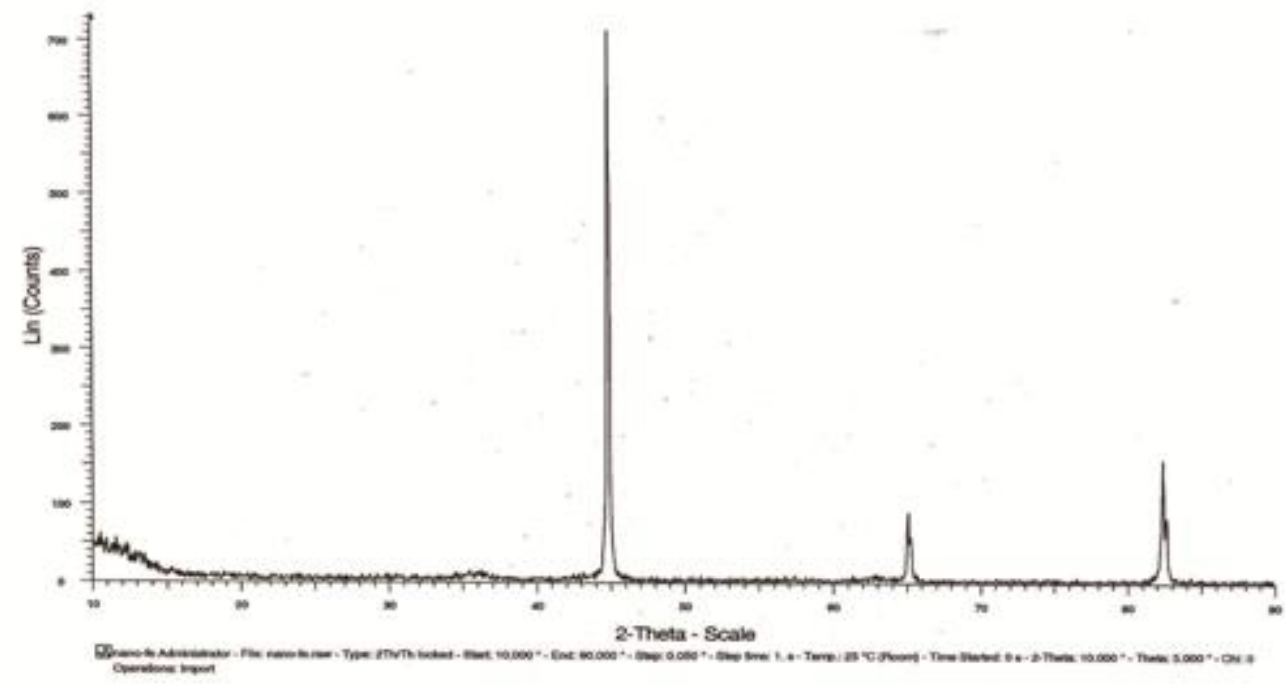

Chart 11. XRD spectra related to iron nanoparticles

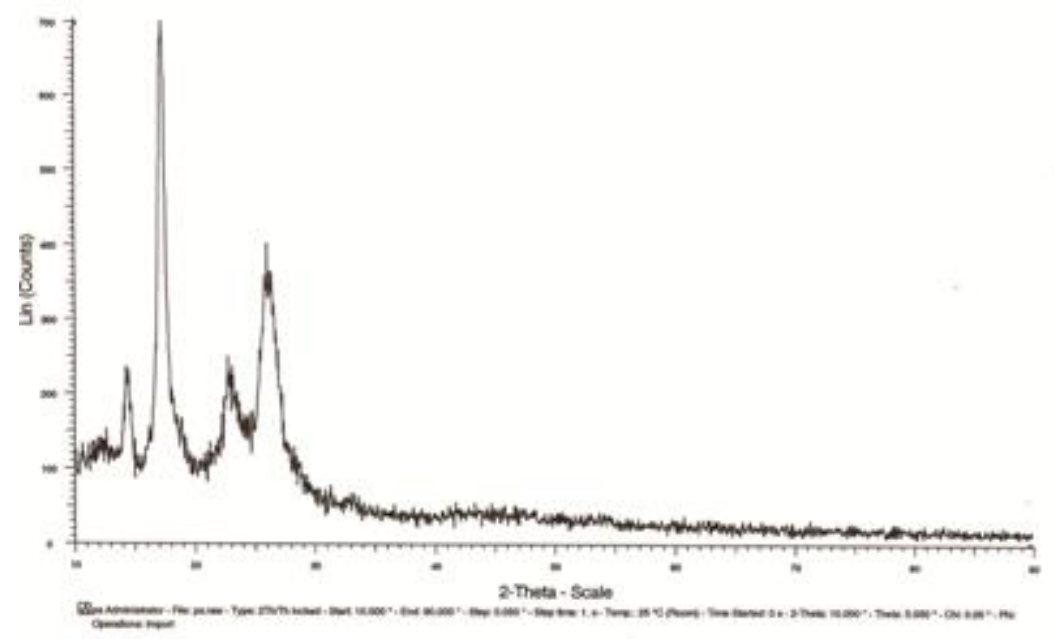

Chart 12. XRD spectra related to membrane PES21\% 


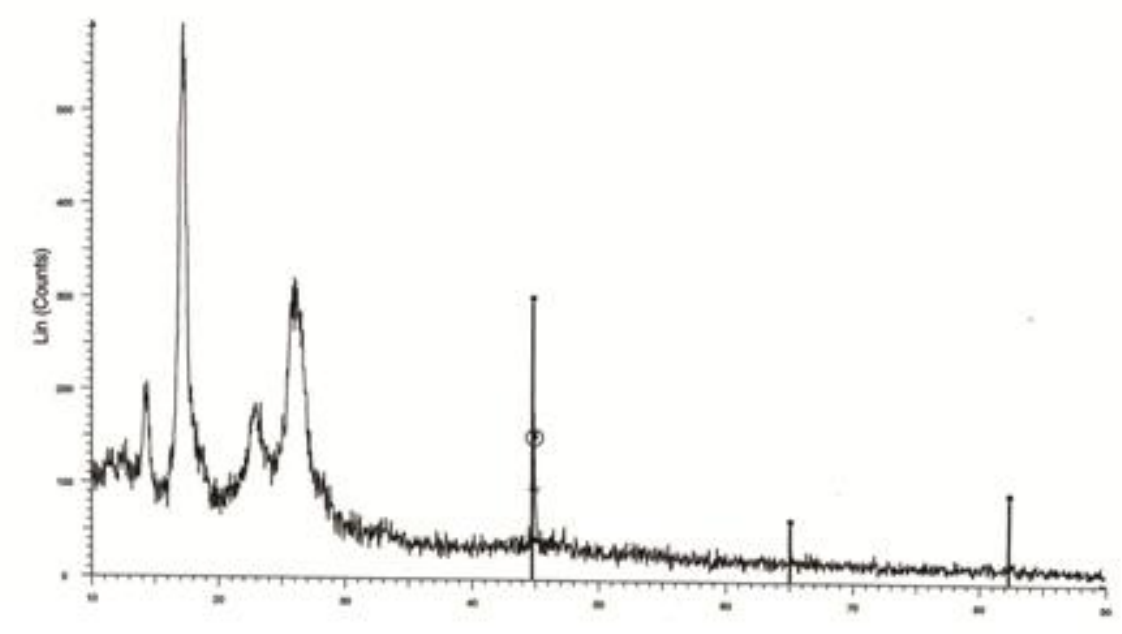

Chart 13. XRD spectra related to membrane Nano $3 \%$ by $21 \%$ PES

\subsubsection{The FTIR spectrum}

In order to ensure the formation of polymerization on the surface this test is performed. We conducted two tests on the most efficient membranes. Polyether sulfone first test was on the membrane of $21 \%$ (Chart 14) and the second test on polyether sulfone membranes with $3 \%$ to $21 \%$ iron nanoparticles (Chart 15). As can be seen in Chart 14, a peak at $\left(\mathrm{cm}^{-1}\right) 1578.79$ that relate to $\mathrm{c}=\mathrm{c}$ aromatic polyether sulfone because aromatic rings and a courier at $\left(\mathrm{cm}^{-1}\right)$ 1720.5 that related to $\mathrm{c}=\mathrm{o}$ str signs are hydrophilic surface and a peak at $\left(\mathrm{cm}^{-1}\right) 2907.01$ that related to simple carbon-hydrogen bond $\mathrm{SP}^{3} \mathrm{CH}$ str symptoms and a peak at $\left(\mathrm{cm}^{-1}\right) 3068.81$ that related to hydrogen-carbon double bond is an indication $\mathrm{SP}^{2} \mathrm{CH}$ str.

In Chart 15 also observed a peak at $\left(\mathrm{cm}^{-1}\right) 1578.14$ that relate to $\mathrm{c}=\mathrm{c}$ aromatic polyether sulfone because aromatic rings and a courier at $\left(\mathrm{cm}^{-1}\right) 1706.41$ that is related to acid level of the peak indicates that an acidic environment is a good condition for the removal of nitrate and we have the highest removal because of the high PH iron hydroxide precipitates. Zerovalent iron is very strong electron donor and the potential for recovery is lower than other metals that's why low PH iron oxides that are positively charged anionic ligands are more attracted to when PH solution is above the isoelectric point and become negatively charged surface oxidation start cleaning surface that can form with cations.

In this shape of a peak in $\left(\mathrm{cm}^{-1}\right) 2906.03$ that related to simple carbon-hydrogen bond $\mathrm{SP}^{3} \mathrm{CH}$ STR is a sign and a peak at $\left(\mathrm{cm}^{-1}\right) 3066.61$ that related to hydrogen-carbon double bond $\mathrm{SP}^{2}$ CH str. [12] 


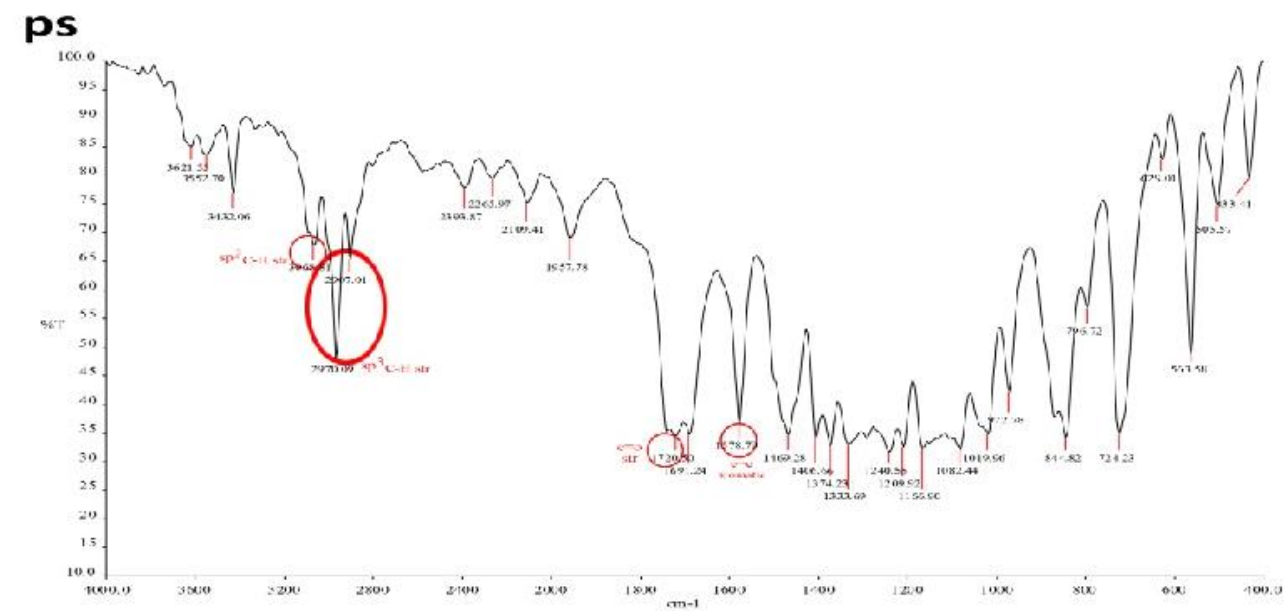

Chart 14. FTIR images related to PES membrane

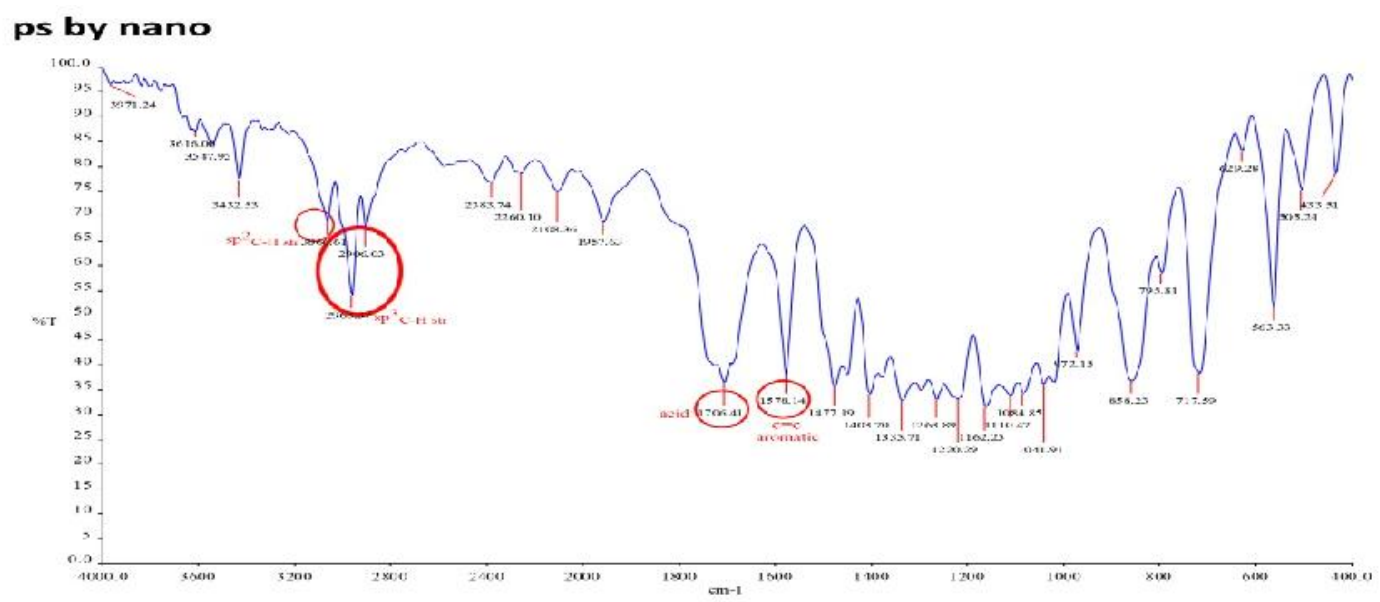

Chart 15. Image FTIR related to membrane PES by Nano

\section{CONCLUSION}

The results of the experiments were obtained as follows:

- By increasing the pressure difference between two sides of the membrane 4 times to 14 times, the separation percentage of nitrate will be reduced, while increasing transmission volume flux.

- With increasing the percentage of polyether sulfone from 15 percent to 21 the percentage of nitrate separation increases, while the volume flux reduced. 
- By adding nanoparticles to the membrane, separation and flux the percentage of at a given operating conditions significantly increased compared to non-Nano particle.

- With increasing the percentage of iron nanoparticles from $3 \%$ to $9 \%$, the percentage of separation decreases as the flux through membrane increases.

- by adding sulfate ions intervention, the percentage of low separation and mass flux through the membrane increases in both cell membranes and membrane iron nanoparticles without nanoparticles significantly reduced.

In the end, the Capillary model, experimental observations were modeled in MATLAB environment and its results indicate the ability of the model to predict the behavior of the system.

\section{ACKNOWLEDGEMENT}

I thank Azad university of Shiraz and respected professor that help me in this investigation.

\section{REFERENCES}

[1] Torabian A, Shokouhi M.M, Gholam R.N. B., Ghadim K.A.A., Safaeifar M. To evaluate the performance of a nano-filtration membrane for the removal of nitrate from drinking water in practical terms, the Journal of Water and Wastewater, Spring., 2007, 18(1), 15-23.

[2] Jahangiri S, Mousavi S.M, Ghaffarian V. Modeling and simulation of isolation and purification of $\beta$ - carotene from carrot seeds using supercritical extraction industries-nanofiltration, the Twelfth National Congress of Iran Chemical Engineering., 2011.

[3] Pinott A, Graiver N, Califano A, Zaritzky N. Diffusion of Nitrite and Nitrate Salts in Pork Tissue in the Presence of Sodium Chloride, J. of Food Science., 2001, 67(6), 2165-2171.

[4] Bhatnagar A, Kumar E, Sillanpaa M. Nitrate removal from water by nanoalumina: Characterization and sorption studies, Chemical Engineering Journal., 2010, 163, 317-323.

[5] http://electronmicroscopy.org/images/fe-sem-microscopy.jpg

[6] Kesting P.S, Toshi S.V, Trivedi J.J, Devmurari C.V, Rao A.P and Ghosh P.K. Probing the structural variations of thin film composite Ro membranes obtained 
by coating polyamide over polysulfone membranes of different pore dimensions. J. memb sci., 2006, 278, 19 -25.

[7] Paugam L, Taha S, Dorange G, Jaouen P, Quéméneur F. Mechanism of nitrate ions transfer in nanofiltration depending on pressure, $\mathrm{pH}$, concentration and medium composition, J. Membr. Sci., 2004, 231(1-2), 37-46.

[8] $\mathrm{Xu} \mathrm{Y}$, and Lebrun R.E. Investigation of the solute separation by charged nanofiltration membrane: effect of $\mathrm{pH}$, ionic strength and solute type, J. Membr. Sci., 1999, 158(1-2), 93-104.

[9] Membrane separation. Author: Amir Hossein Setude Bidokhti, Wendsday, 27 farvardin, 1390.

[10]Hosseini S.M, Kholghi M. ,Ataii A.B., Bagheri M.M.M. Labratury study of decreasing nitrate from drinkable water by using bimetal iron copper nanoparticle.hygin and heath magezine ,ordibehesht., 1390, 1, 94-103.

[11] Azauli M.A, Dianat R.A, Safarpur M. Nitrate eliminiation from water by zero valancy iron nanoparticle in presence of ultraviolet ray and without it, Medical science university Magezine of mazandaran, khordad 93, period., 24(13), 151161.

[12] http://www.science-and-fun.de/tools/

How to cite this article:

Shojaeian M.J and Kamayab Moghadas B. Polyether sulfone membrane modeling and construction for the removal of nitrate from water using ion interference sulfate and iron nano-particle. J. Fundam. Appl. Sci., 2016, 8(2S), 31-51. 